\title{
Producción de Ácidos Grasos Poliinsaturados a partir de Biomasa Microalgal en un Cultivo Heterotrófico
}

\author{
Production of Polyunsaturated Fatty Acids from \\ Microalgal Biomass in Heterotrophic Culture
}

\section{Produção de Ácidos Gordos Poli-Insaturados a partir de Biomassa de Microalgas na Cultura Heterotróficos}

\author{
Gloria Inés Leal Medina1; José Eduardo Abril Bonett'; Silvia Juliana Martínez Gélvez'; Yaneth Amparo \\ Muñoz Peñaloza ${ }^{2}$; Elena María Peñaranda Lizarazo; Néstor Andrés Urbina Suárez ${ }^{2^{*}}$ \\ ${ }^{1}$ Ingeniería Biotecnológica. Universidad Francisco de Paula Santander (UFPS), \\ Avenida Gran Colombia N ${ }^{\circ} 12 \mathrm{E}-96$ Colsag, Cúcuta, Colombia \\ ${ }^{2}$ Departamento de Medio Ambiente. Universidad Francisco de Paula Santander (UFPS), \\ Avenida Gran Colombia N²12E-96 Colsag, Cúcuta, Colombia \\ *nestorandresus@ufps.edu.co
}

Fecha Recepción: 24 de mayo de 2016 Fecha Aceptación: 26 de septiembre de 2016

\begin{abstract}
Resumen
El trabajo aquí presentado se enfocó en la producción de ácidos grasos poliinsaturados o PUFA's (por sus siglas del inglés PolyUnsaturated Fatty Acids) a partir de biomasa microalgal en un cultivo heterotrófico. Para esto, se utilizaron las algas Chlorella sp. y Scenedesmus sp., en condiciones heterotróficas, posteriormente se seleccionó la cepa con mayor productividad, se realizaron las cinéticas con ambas algas para cuantificar la concentración de biomasa, glucosa, nitrógeno y fósforo; se extrajeron los lípidos y se analizaron por cromatografía de gases. El cultivo heterotrófico se estableció en un reactor de tanque agitado de flujo continuo o CSTR (por sus siglas del inglés Continuous Stirred Tank Reactor) de 1L, con las siguientes condiciones; $28^{\circ} \mathrm{C}, 1 \mathrm{vvm}, \mathrm{pH} 6,8$ y relación $\mathrm{C} / \mathrm{N} 12: 1$. Luego, se realizó el cultivo en un "Biorreactor BioFlo 115" con volumen de 10L y se determinó la productividad de los lípidos obtenidos. El perfil lipídico permitió establecer que el ácido graso obtenido en mayor cantidad en CHL2 es el ácido oleico (C 18:1) con un porcentaje igual al 28,75 del total de ácidos grasos, también se destacan la acumulación de los ácidos grasos palmitoléico (C 16:1) con 19,75\%, ácido araquídico (C 20:0) con $19,37 \%$, ácido linoleico (C 18:2) con 11,86\%, ácido palmítico (C 16:0) con 7,24\%, ácido linolénico ( $\gamma$-C 18:3) con 2,61\%, ácido erúcico (C 22:1) con 4,61\% y ácido esteárico (C 18:0) 2,4\%.
\end{abstract}

Palabras clave: ácidos grasos poliinsaturados (PUFA's), bomasa microalgal, Chlorella sp., Scenedesmus sp.

\begin{abstract}
In this research, we obtained Polyunsaturated Fatty Acids (PUFAs) from Microalgal Biomass in heterotrophic culture. In order to obtain these PUFA's we used Chlorella sp. and Scenedesmus sp. strains with heterotrophic conditions. Subsequently, we selected the strain with higher productivity and determined the growth kinetics and biomass, glucose, phosphorus and nitrogen yields and the lipids extracted analyzed by gas chromatography. The heterotrophic culture was established in $1 \mathrm{~L}$ Continuos Stirred-Tank Reactor (CSTR) under specific conditions; $28^{\circ} \mathrm{C}, 1 \mathrm{vvm}, \mathrm{pH} 6,8$ and a relation $\mathrm{C} / \mathrm{N} 12: 1$. Then, we performed sub-cultivation in a "Bioreactor BioFlo115" with 10L volume and made productivity analysis of lipids. Lipids profile allowed us to determine that higher Fatty Acid present in Chlorella sp. is oleic acid (C 18:1) and represented a percentage of 28,75 of total fatty acids. Also we noted the accumulation the
\end{abstract}

Cita: Leal GI, Abril JE, Martínez SJ, Muñoz YA, Peñaranda EM, Urbina NA. Producción de Ácidos Grasos Poliinsaturados a partir de Biomasa Microalgal en un Cultivo Heterotrófico. rev.ion.2017;30(1):91-103. 
other fatty acids like; palmitoleic acid (C 16:1) 19.75\%, arachidonic acid (C 20:0) 19.37\%, linoleic acid (C 18:2) 11.86\%, palmitic acid (C 16:0) 7.24\%, linoleic acid ( $\gamma-C$ 18:3), 2.61\%, erucic acid (C 22:1) 4.61\%, stearic acid (C 18:0) 2.4\%.

Keywords: PUFA's, microalgal biomass, Chlorella sp., Scenedesmus sp.

\section{Resumo}

O trabalho aqui apresentado voltada para a produção de ácidos graxos poliinsaturados ou PUFAs (por sua sigla em ácidos graxos poliinsaturados Inglês) a partir da biomassa de microalgas em uma cultura heterotróficos. Para isso, foram utilizados algas Chlorella sp. e Scenedesmus sp., em condições heterotróficas, em seguida, a tensão com o aumento da produtividade foi seleccionado, as cinéticas foram realizadas tanto com as algas para quantificar a concentração de biomassa, da glicose, de azoto e de fósforo; lípidos foram extraídos e analisados por cromatografia em fase gasosa. A cultura foi criada heterotrófica em um reactor de tanque agitado de fluxo contínuo (CSTR) de 1L, com as seguintes condições; $28^{\circ} \mathrm{C}, 1 \mathrm{vvm}, \mathrm{pH} 6,8$ e $\mathrm{C} / \mathrm{N}$ rácio de $12: 1$. Em seguida, a cultura foi realizada num "Biorreactor BioFlo115" com um volume de 10L e produtividade de lípidos obtidos foi determinada. O perfil lipídico estabelecido que o ácido graxo obtido em maiores quantidades em CHL2 é ácido oleico (C 18:1), com uma percentagem igual a 28,75 do total de ácidos graxos, também destaca a acumulação de ácidos graxos palmitoléico (C 16:1), com 19,75\%, ácido araquídico (C 20:0), com 19,37\%, ácido linoleico (C 18:2) $11,86 \%$, ácido palmítico (C 16:0) $7,24 \%$, ácido linolénico $(\gamma-C 18: 3)$ com $2,61 \%$ de ácido erúcico (C 22:1) com 4,61\% de ácido esteárico e (C 18:0) 2,4\%.

Palabras-chave: ácidos graxos poliinsaturados (PUFAs), biomassa de microalgas, Chlorella sp, Scenedesmus sp.

\section{Introducción}

El ácido linoleico y el ácido a-linolénico, son Ácidos Grasos Polilnsaturados (AGPI o PUFA's) esenciales, pertenecientes a los grupos omega-6 y omega-3 respectivamente; éstos son precursores de otros AGPI como el ácido araquidónico, el ácido eicosapentaenoico (EPA), y el ácido docosahexaenoico (DHA), los cuales están asociados con la prevención de enfermedades cardiovasculares, cáncer y el correcto funcionamiento cerebral [1].

El carácter esencial de estos compuestos y su importancia fisiológica se traducen en una demanda creciente y la búsqueda de estrategias para incrementar su consumo. El aceite de pescado es la principal fuente de AGPI, sin embargo, se han asociado con varias desventajas como son: presencia de olores desagradables, contaminación con metales pesados, presencia de colesterol y una composición variable que dificulta la purificación $[2,3]$. Como los peces obtienen los AGPI del Zooplancton, el cual se alimenta de algas, muchos esfuerzos se han enfocado en el desarrollo de tecnologías que permitan producir estos ácidos grasos directamente de las microalgas [3].

Las microalgas son un grupo altamente diverso de organismos unicelulares entre los que se encuentran protistas eucarióticos y cianobacterias procarióticas, que pueden crecer rápidamente debido a su estructura simple [4]. Tal como se relaciona anteriormente, las microalgas al poseer una fracción lipídica rica en AGPI han sido objeto de estudios de bioprospección y de numerosas investigaciones que buscan optimizar sus condiciones de cultivo y producción, así como reconocer sus ventajas.

Se infiere que la producción de aceite a partir de seres unicelulares es un concepto relativamente nuevo [5], y las microalgas se muestran como una opción prometedora, ya que resulta ser más económica que las fuentes actuales de estos compuestos. Además, se ha reportado que el contenido lipídico de la biomasa de microalgas puede variar, tal es el caso de: Ankistrodesmus sp. (263,6mg/g biomasa seca), A. nannoselene (316 mg/g biomasa seca) y Scenedesmus sp. (243,3mg/g biomasa seca) en cultivos con medios sin nitrógeno. Por otras parte para el caso de Scenedesmus quadricauda y Chlorella sp., presentaron la mayor producción de biomasa seca con 159,1 y 221,1 mg/g biomasa seca, respectivamente, en medios con nitrógeno [6].

El contenido de lípidos de las microalgas se ve influenciado por diferentes factores en los que se desarrollan los cultivos, los cuales afectan la producción relativa de ácidos grasos y su contenido total. Muchos procesos de producción basados en crecimiento autotrófico se ven obstaculizados por la 
limitación de luz que producirían por tanto bajas tasas de crecimiento y bajas densidades celulares [3]

Se ha considerado entonces, que la producción de AGPI a partir de microalgas puede ser una alternativa más económica, especialmente bajo condiciones de heterotrofia, porque se reducen los costos de producción. Se ha relacionado que la producción de EPA y DHA es más alta en cultivos bajo oscuridad en especies como las diatomeas Tetraselmis spp., N. laevis y $N$. alba, además se ha demostrado que los sistemas de producción heterotrófica pueden exhibir una productividad de AGPI omega-3 de 2 o 3 órdenes de magnitud por encima de las obtenidas bajo autotrofía [7]. Tomando en cuenta lo anteriormente expuesto, el presente estudio evaluó la producción de ácidos grasos poliinsaturados de un cultivo de microalgas en condiciones de heterotrofia.

\section{Metodología}

\section{Activación de las cepas de microalgas}

Se utilizaron cepas de Scenedesmus sp. y Chlorella sp., provenientes del Banco de Cepas del Laboratorio de Bioprocesos de la Universidad Francisco de Paula Santander, estas cepas se aislaron mediante la utilización de medios selectivos y se encuentran caracterizadas morfológicamente, las mismas cepas han sido usadas y reportadas previamente [8]. En otro reporte estas mismas cepas se cultivaron en medio PCG en condiciones de autotrofía y se activaron a $30^{\circ} \mathrm{C}$, con una intensidad lumínica de $200 \mu \mathrm{mol} / \mathrm{m}^{2} \mathrm{~s}$ (provista por lámparas fluorescentes de luz fría de día), una agitación de 125rpm, 1vvm y un $\mathrm{pH}$ de 8 para Scenedesmus sp. y de 6,8 para Chlorella sp. [9]

\section{Acondicionamiento de las cepas de microalgas a heterotrofia}

Una vez activadas las cepas, se procedió a su adaptación a condiciones de heterotrofía (crecimiento en ausencia de luz y utilizando un sustrato orgánico). Se utilizó el medio PCG modificado [10], el cual contiene glucosa en una relación 12:1 con respecto a la fuente de nitrógeno. En esta etapa se evaluó la producción de biomasa y de lípidos para luego seleccionar la cepa más productiva. Las condiciones de cultivo para esta etapa fueron: agitación a 150rpm, 1vvm de caudal de aire, pH 8 para Scenedesmus sp. y pH 6,8 para Chlorella sp., a $30^{\circ} \mathrm{C}$.
Cinética de crecimiento a escala de $10 \mathrm{~L}$

Una vez seleccionada la cepa, se procedió a realizar la cinética en el Biorreactor BioFlo115. Se utilizó la consola de control y se adaptó un recipiente a capacidad de $15 \mathrm{~L}$, con el fin de tener un volumen operacional de 10L. La cepa se inoculó en el medio PCG modificado [10], al 10\% del volumen. Las condiciones fueron de agitación a $150 \mathrm{rpm}$, caudal de aire de 1,2vvm, temperatura $28^{\circ} \mathrm{C}$ y pH de 6,8 . Se realizó medición de biomasa, consumo de nutrientes y determinación final de lípidos al concluir la cinética.

\section{Métodos de análisis}

Para analizar la cinética de crecimiento de $10 \mathrm{~L}$ se determinó la producción de biomasa, el consumo de nitrógeno, fósforo y glucosa. La biomasa se cuantificó en peso seco mediante filtración al vacío usando membranas de nitrocelulosa de $0,22 \mu \mathrm{m}$ de diámetro de poro (Millipore ${ }^{\circledR}$ ) [10]. La cuantificación de nitratos se realizó por el método de Brucina-Ácido Sulfámico midiendo posteriormente la absorbancia a 410nm [11]. La cuantificación de fósforo inorgánico se realizó a 660nm por el método modificado de Taussky y Shorr como establece Perales H [9]. Para determinar el consumo de glucosa se empleó el método DNS [10].

\section{Extracción y cuantificación de lípidos}

La biomasa fue floculada utilizando Cloruro de aluminio $\left(\mathrm{AlCl}_{3}\right)$ en una concentración de $40 \mathrm{~g} / \mathrm{L}$ en una dosis de $1,5 \mathrm{~mL}$ de floculante por cada $100 \mathrm{~mL}$ de medio. Posteriormente, fue secada en el liofilizador Labcom y pulverizada en mortero. Finalmente, se extrajeron los lípidos, con un solvente en un extractor Soxhlet [8].

La cuantificación y determinación del perfil lipídico se realizó siguiendo la metodología propuesta por Sánchez et al. [12]. Los ésteres metílicos resuspendidos en $1 \mathrm{~mL}$ de $\mathrm{h}$-hexano fueron analizados en un cromatógrafo de gases marca: Thermo scientific, modelo: Trace 13007 890A, equipado con un detector modular de ionización de llama (FID) (IEC) y una columna capilar TR-5MS, $30 \mathrm{~m}, 0,25 \mathrm{~mm}$ ID, $0,25 \mu \mathrm{m} \mathrm{HP}-5$ con un gradiente de temperatura de $10^{\circ} \mathrm{Cmin}^{-1}$ desde 125 hasta $255^{\circ} \mathrm{C}$ y $270^{\circ} \mathrm{C}$ por $3 \mathrm{~min}$. Se empleó Hidrógeno como gas portador, manteniendo un flujo de $1 \mathrm{mLmin}^{-1}$ El volumen de inyección fue de $1 \mu$ en modo Split 100:1. La temperatura del inyector y del detector fue de $250^{\circ} \mathrm{C}$. 


\section{Análisis de datos}

Todos los experimentos se realizaron por triplicado. Los resultados fueron verificados estadísticamente por la prueba $\mathrm{T}$ de Student $(P<0,05)$ utilizando el programa Sigma-Plot-Stat versión 14.

\section{Resultados y Discusión}

Acondicionamiento de las cepas de Chlorella sp. y Scenedesmus sp., a heterotrofia y cinética de crecimiento a escala de $1 \mathrm{~L}$

Las cepas de Chlorella sp. (CHL2) y Scenedesmus $\mathrm{sp}$. (SCE) crecieron satisfactoriamente en medio sólido PCG modificado, sin embargo el tiempo necesario para evidenciar crecimiento en caja Petri fue mayor para SCE (19 días) en comparación con CHL2 (12 días).

Dentro del diverso grupo de las microalgas, las pertenecientes a la familia de las algas verdes en su gran mayoría pueden crecer bajo condiciones heterotróficas. De hecho, algunas especies de microalgas presentan mayor velocidad de crecimiento $[13,14]$ y productividad de lípidos
$[15,16]$ en condiciones heterotróficas [17]. En el caso de Chlorella se han reportado varios estudios del comportamiento bajo este régimen nutricional, $[18,19]$ y se ha logrado alcanzar valores de ácidos grasos de hasta 3 órdenes de magnitud por encima de los alcanzados en autotrofía [20]. Para el caso de Scenedesmus, son muy pocos los trabajos bajo condiciones de heterotrofía, se reporta que la productividad de biomasa de este género de microalgas puede ser 2,5 veces mayor en comparación al cultivo autotrófico.

Los resultados de la producción de biomasa y consumo de nutrientes de las cepas se muestran en la Figura 1, donde se aprecia que el crecimiento de la cepa CHL2 fue relativamente mayor $(1,2 \pm 0,0141 \mathrm{~g} / \mathrm{L})$ en comparación a la máxima alcanzada por la cepa SCE $(0,8200 \pm 0,0235 \mathrm{~g} / \mathrm{L})$. Como se puede observar ocurre un descenso de biomasa a partir de la hora 150 lo cual se debe a que la cantidad de sustrato disponible para este tiempo de la cinética es prácticamente nula, lo que evidencia que el número de células que mueren es mayor al número de células que se generan.

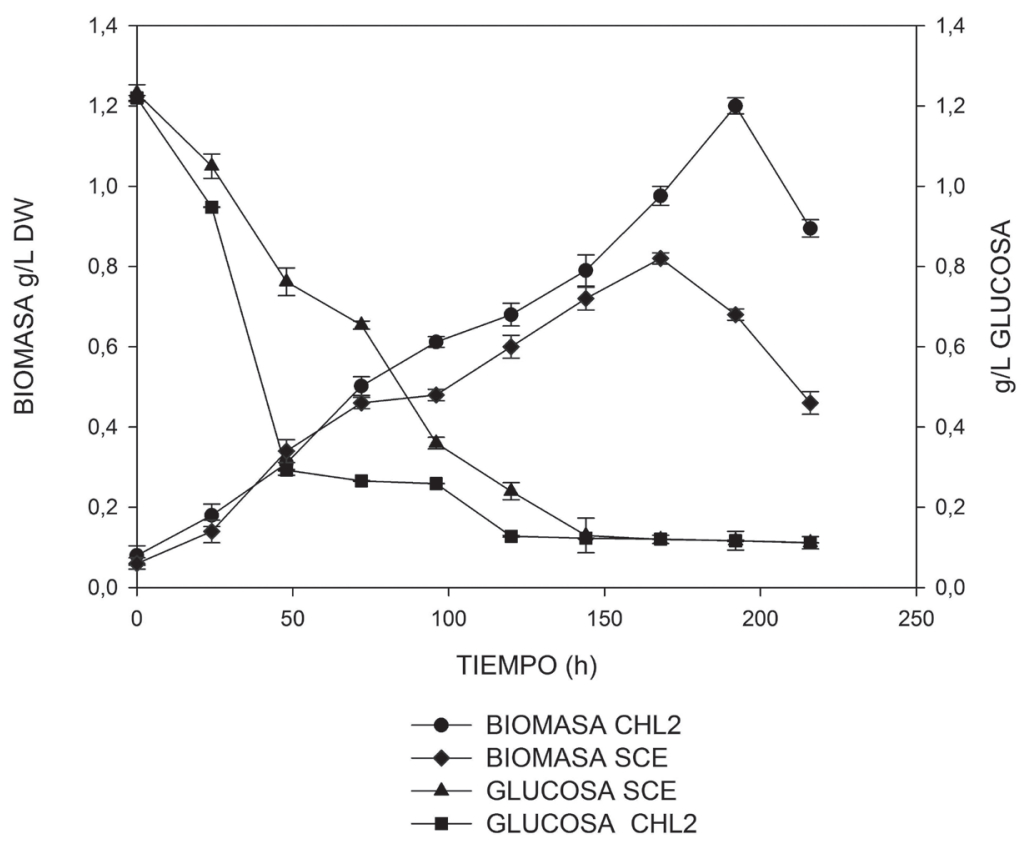

Figura 1. Cinética de Crecimiento CHL2 y SCE.

La mayor parte de las algas verdes pueden crecer heterotróficamente en oscuridad, lo que supone que estos organismos poseen la capacidad de obtener a expensas de un sustrato orgánico, en este caso glucosa, la energía y el carbono necesario para la síntesis celular. Para el caso de Chlorella varios autores han reportado crecimiento de esta alga bajo condiciones de heterotrofia; así mismo, se ha reportado que al cultivar células de Chlorella zofingiensis con $30 \mathrm{~g} / \mathrm{L}$ de glucosa en la oscuridad, se obtiene un rendimiento mayor de biomasa $(41 \%)$, lípidos totales $(98 \%)$ y una productividad 
de lípidos de $354 \mathrm{mg} / \mathrm{Ld}$ equivalente a $91 \%$ en comparación con el cultivo foto autotrófico [14].

De igual forma, se reportan las ventajas de utilizar células heterótrofas como inóculo de un cultivo fotoautotrófico en estanques abiertos para la producción a gran escala de biomasa con alto contenido de lípidos de Chlorella sorokiniana; adicionalmente compararon el desempeño de cultivos fotoautotróficos y heterotróficos encontrando que la velocidad de crecimiento, la densidad celular y la productividad fue 7,4 veces mayor bajo condiciones heterotróficas [21].

En relación a especies del género Scenedesmus, son pocos los trabajos reportados, no obstante se encontró para Scenedesmus incrassatulus una producción de biomasa de 1,21g/L, mayor a la alcanzada en condiciones de autotrofía [9]. Sin embargo, en la presente investigación, la producción de biomasa fue menor, lo cual se podría explicar con el hecho que a diferencia del trabajo referido, no se utilizó extracto de levadura.

Otros trabajos reportan valores de biomasa para Chlorella sp. $(0,88 \mathrm{~g} / \mathrm{L})$ y Scenedesmus sp. $(0,68 \mathrm{~g} / \mathrm{L})$ en condiciones de autotrofía [22] por debajo de los alcanzados en este trabajo.

Para la condición de heterotrofia algunos investigadores sugieren que especies de los géneros Scenedesmus y Chlorella son capaces de utilizar la glucosa probablemente, a través de la vía de las pentosas fosfatos del ciclo EmbdenMeyerhof-Parnas [10,23], ya que la glucosa-6fosfato deshidrogenasa, la primera enzima de esta vía, tiene una alta actividad en las células de estos géneros [10]. Existen reportes que en Scenedesmus obliquus la densidad celular inducida por la glucosa puede ser 3,1-3,6 veces mayor que la inducida por la luz u otro tipo de sustratos orgánicos [24]. Esto se explicaría dado que la energía obtenida a partir de la glucosa por una mol de sustrato produce 38 moles de ATP, lo que es una ganancia mayor a la alcanzada por la fotosíntesis donde se alcanzan 18 moles de ATP [23]. Los resultados obtenidos en esta etapa con relación a la producción de biomasa, mostraron que la cepa CHL2 se adaptó mejor a las condiciones de heterotrofia en comparación con el resultado mostrado por la cepa SCE.

La Figura 2 muestra la concentración de lípidos totales alcanzados tanto para la cepa CHL2, como para la cepa SCE en condiciones de heterotrofia, al igual que un control en condiciones de autotrofía para cada cepa. Se observa, que el mayor contenido de lípidos [mg de lípido/g de biomasa seca] se alcanzó con la cepa CHL2 en condiciones de heterotrofía $(150,4 \pm 2,3)$, seguido de la cepa CHL2 en condiciones de autotrofía $(92,5 \pm 4,625)$, para el caso de la cepa SCE el porcentaje más alto se presentó en la condición heterotrófica $(62,4 \pm 0,823)$ en comparación con la condición autotrófica $(51,3 \pm 4,0)$. De acuerdo a los resultados obtenidos, se puede evidenciar que la condición heterotrófica favoreció a la cepa CHL2, perteneciente al género Chlorella en comparación a la cepa SCE perteneciente al género Scenedesmus.

Se ha reportado para Chlorella protothecoides un $55 \%$ de lípidos en condiciones heterotróficas en un medio con acetato de sodio enriquecido con un hidrolizado de maíz [20]. Así mismo, para un cultivo de $2 \mathrm{~g} / \mathrm{L}$ de glucosa y utilizando una cepa de Chlorella sp. de agua dulce, se alcanzó una concentración de $13 \%$ de lípidos en peso seco [25]. Y para el caso de Chlorella vulgaris se alcanzó un porcentaje entre $14 \%-28 \%$ de lípidos en condiciones de heterotrofia utilizando alrededor de $3 \mathrm{~g} / \mathrm{L}$ de glucosa [26].

Las condiciones de cultivo influyen en la producción de biomasa y acumulación de lípidos [27], el hecho de crecer en ausencia de luz y con sustrato orgánico permite que exista un menor gasto energético en la síntesis de lípidos; además se puede controlar la velocidad de crecimiento en condiciones de ausencia de luz en comparación con procesos fotoautotróficos, la razón radica en que en condiciones de heterotrofia es el sustrato orgánico, quien ejerce un papel fundamental en la síntesis tanto de biomasa como de lípidos, cuando la velocidad de crecimiento disminuye, adicionar más sustrato al medio permite mantener una síntesis de lípidos. Caso contrario, con el proceso fotoautotrófico donde la luz pasa a ser el aspecto que ejerce el control sobre el proceso. El problema radica en que a medida que se aumenta la luz, aumenta la densidad celular, llegando a un punto donde el proceso de autosombreado de las células limita el crecimiento y por ende la síntesis de ácidos grasos, así se aumente la intensidad de luz, si el cultivo presenta una alta densidad celular, no es posible mantener un control en la velocidad de crecimiento. Este proceso podría explicar lo obtenido en este trabajo, donde ambas cepas obtuvieron una mayor cantidad de lípidos en condiciones heterotróficas. 


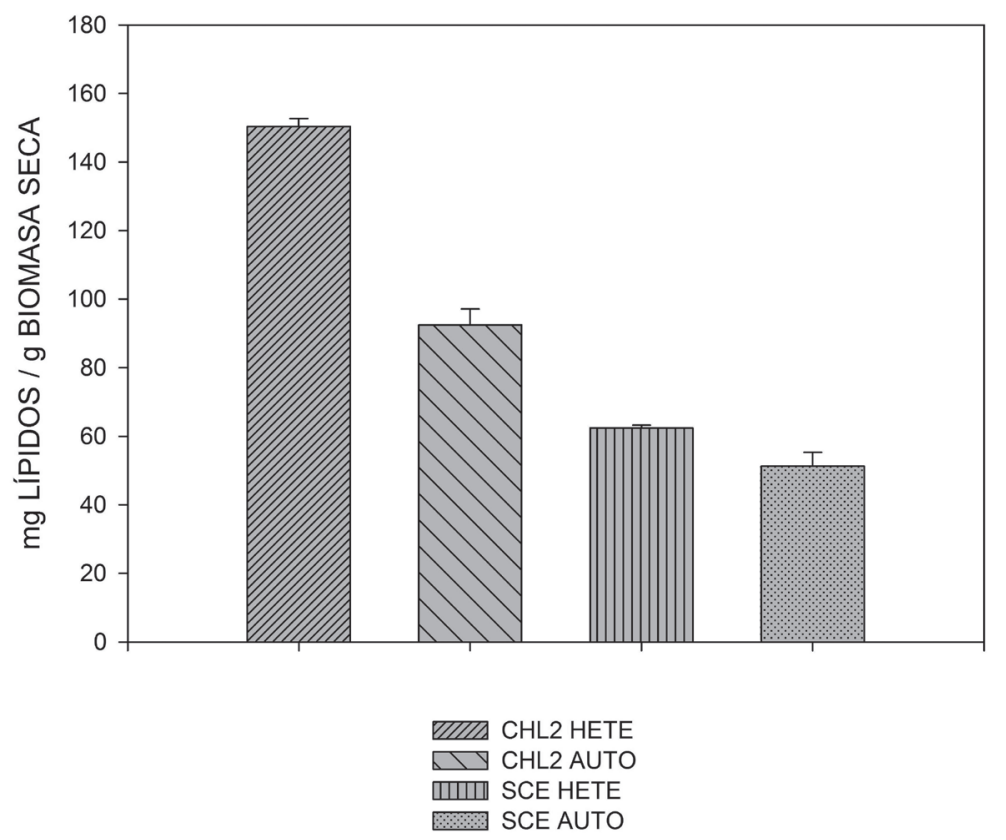

Figura 2. Contenido de lípidos en [mg de lípido/g de biomasa seca] de las cepas CHL2 y SCE en condiciones heterotróficas y autotróficas

La cepa CHL2 perteneciente al género Chlorella $\mathrm{sp}$., se seleccionó para realizar la cinética de crecimiento a escala de $10 \mathrm{~L}$ ya que presentó mejores resultados en términos de biomasa y de lípidos en comparación con la cepa SCE perteneciente al género Scenedesmus sp.

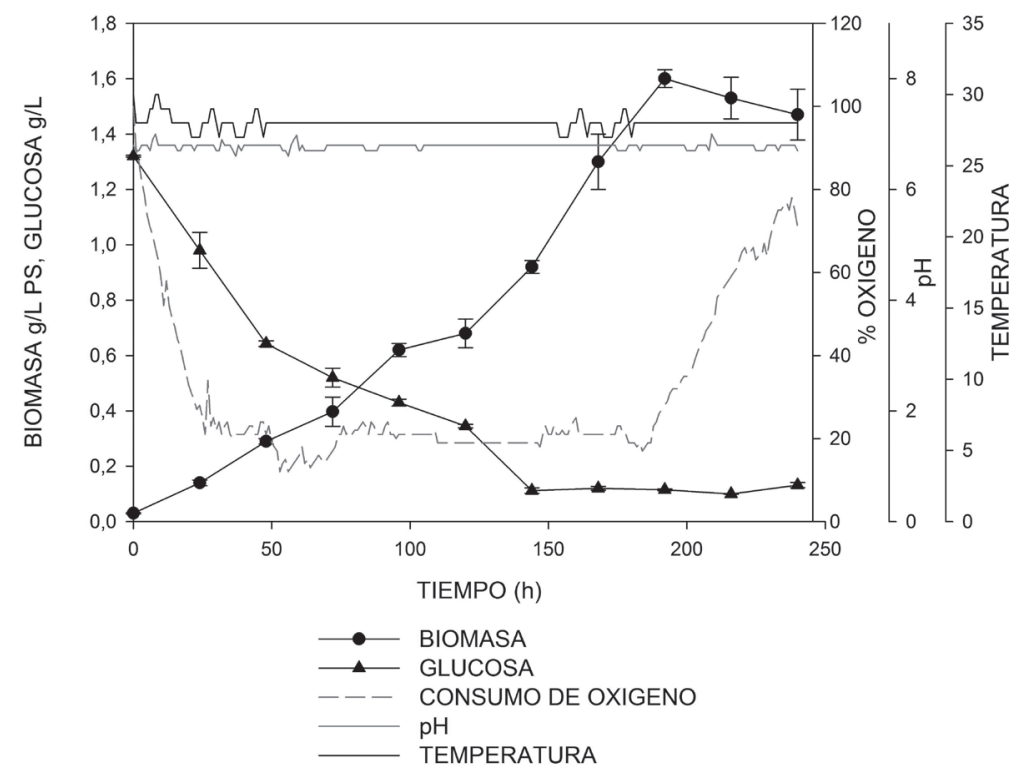

Figura 3. Cinética de crecimiento de CHL2 en condiciones heterotróficas escala 10L. 
Cinética de crecimiento en el BioFlo115 a escala 10L

En la Figura 3 se puede apreciar que la máxima concentración de biomasa $(1,6 \pm 0,032 \mathrm{~g} / \mathrm{L})$ alcanzada en el proceso fue en la hora 180 , después de ese tiempo el sistema entró en estado de latencia, donde ya no se evidenció un consumo de sustrato; lo cual se demuestra con una baja sustancial en el consumo de oxígeno durante las primeras $24 \mathrm{~h}$ del proceso, después de los cuales este valor se mantuvo constante hasta la hora 180. Lo anterior permite inferir que la baja tasa en el consumo de oxígeno se presenta porque no hay requerimiento para crecimiento celular y el poco oxígeno que se consume, se utiliza para actividades de mantenimiento. En cuanto el $\mathrm{pH}$ y la temperatura se puede evidenciar que se mantuvieron relativamente constantes y que el sistema de control actuó satisfactoriamente durante la cinética de crecimiento.

En la actualidad, varias investigaciones en biotecnología de microalgas se enfocan en la obtención de cepas con alto contenido de ácidos grasos poliinsaturados, especialmente; ácido eicosapentaenoico (EPA), araquidónico (AA) y docosahexaenoico (DHA) [28]. Las microalgas con contenidos lipídicos altos, han tomado un gran interés para diversos usos, por lo anterior la cantidad de lípidos contenidos en la biomasa, la velocidad de crecimiento, la asimilación de nutrientes y la tolerancia a condiciones ambientales constituyen parámetros de gran importancia $[29,30]$.

Los resultados aquí obtenidos sobre el contenido de lípidos, corresponden a la cinética realizada en un biorreactor de 10L. En la Figura 4 se relaciona el contenido de lípidos con la concentración máxima de biomasa en peso seco. Se reporta que el contenido lipídico más alto en la cepa de Chlorella sp., se presentó en cultivo heterotrófico con un valor de 158,7 $\pm 3,87$ mglípidos/gbiomasa seca para el caso del cultivo autotrófico (control) se alcanzó un contenido

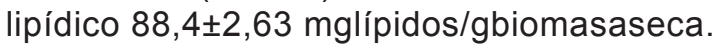

Las microalgas del género Chlorella han sido ampliamente estudiadas, existen reportes de Chlorella protothecoides donde se ha demostrado en cultivos con glucosa y bajas concentraciones de nitrógeno, valores de lípidos 3,4 veces mayores que en condiciones foto autotróficas [17-19].

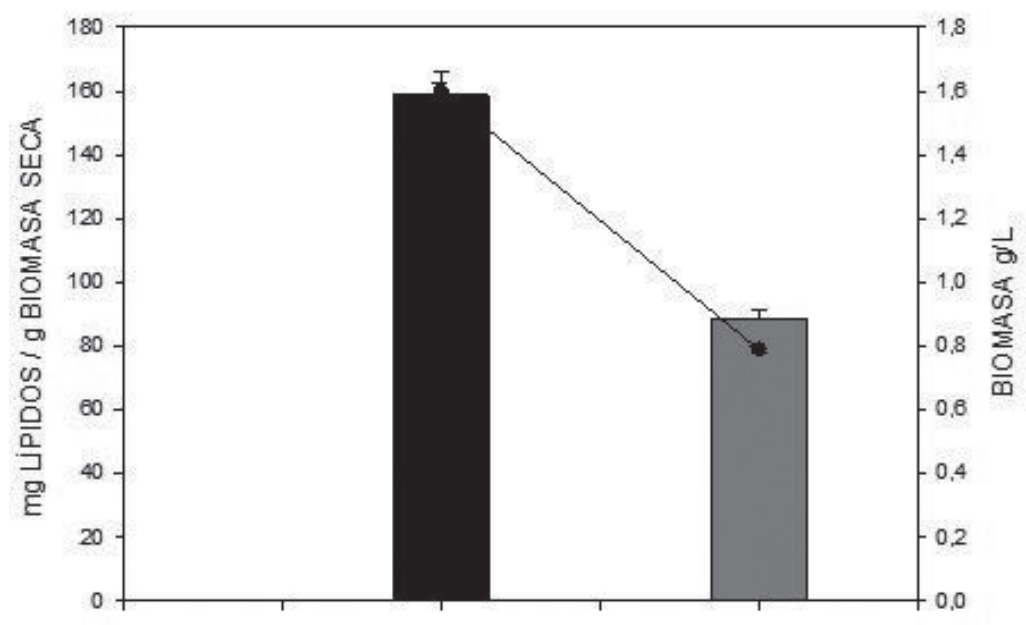

Figura 4. Porcentaje de lípidos CHL2 en cinética de 10L. 
Otros autores, han encontrado que el contenido lipídico de Chlorella sp., en condiciones de heterotrofia puede oscilar entre un 10 a $30 \%$ en peso seco [25]; Cuando se aumenta la concentración de glucosa hasta $10 \mathrm{~g} / \mathrm{L}$, existe un aumento considerable de la productividad de lípidos [13], pero se debe tener en cuenta que concentraciones arriba de $25 \mathrm{~g} / \mathrm{L}$ pueden ocasionar una inhibición por sustrato y por ende una disminución en la concentración de biomasa, afectando directamente la acumulación y producción de lípidos. Cabe resaltar De acuerdo a los resultados de este trabajo se confirmó que el cultivo heterotrófico induce la acumulación de lípidos en comparación al cultivo autotrófico, siendo dos veces más alta, como se observa en la Figura 4. Este aumento se explica dado que la concentración de lípidos es directamente proporcional al contenido de biomasa y esta cantidad de biomasa a su vez, incrementa en condiciones de heterotrofia. Además, tomando en cuenta que al no tener luz los procesos fotosintéticos bajan su intensidad, el consumo de sustrato estará relacionado mayoritariamente en actividades de crecimiento permitiendo que más átomos de carbono estén disponibles para la síntesis de lípidos.

Los parámetros cinéticos se reportan en la Tabla 1; la velocidad de crecimiento se calculó en la fase exponencial del cultivo, la productividad de biomasa y lípidos se determinó hasta la concentración máxima alcanzada. La concentración de biomasa (base seca) presentó el valor más alto en el cultivo heterotrófico $(1,6 \pm 0,032 \mathrm{gPS} / \mathrm{L})$ en comparación con el cultivo autotrófico $(0,78 \pm 0,01 \mathrm{gPS} / \mathrm{L})$. La velocidad de crecimiento más alta se presentó en el cultivo heterotrófico obteniéndose un valor de $0,33 \mathrm{~d}^{-1}$, en comparación al cultivo autotrófico $\left(0,16 \mathrm{~d}^{-1}\right)$. De acuerdo a los resultados obtenidos es evidente que la tasa de crecimiento es aproximadamente dos veces mayor en el cultivo heterotrófico. Según Jacob-Lopes (2009) [31], los cultivos autotróficos son dependientes del periodo de exposición a la luz, siendo por tanto, el incremento de la biomasa directamente proporcional al aumento del fotoperiodo. De igual forma, se ha reportado que la intensidad lumínica se puede afectar por diversos factores, entre ellos el aumento de la concentración celular que provoca efectos de sombreados y la formación de zonas oscuras e iluminadas que afectan, los procesos metabólicos [32]. En el cultivo heterotrófico la disponibilidad de sustrato viene dada por la adición de la fuente orgánica por lo tanto, la tasa de crecimiento está sujeta a la disponibilidad del sustrato en el medio, a diferencia del cultivo autotrófico la microalga no requiere realizar procesos fotosintéticos para la formación de los azúcares simples ya que estos se proporcionan al medio. Lo anterior permite explicar la diferencia de la velocidad de crecimiento del cultivo autotrófico con el heterotrófico.

Tabla 1. Parámetros cinéticos cepa CHL2 en heterotrofia.

\begin{tabular}{ccc}
\hline Parámetro & Control & CHL2 HETE \\
\hline Concentración Max. Biomasa $(\mathrm{g} / \mathrm{L})$ & $0,78 \pm 0,01$ & $1,6 \pm 0,032$ \\
$\mu\left(\mathrm{d}^{-1}\right)$ & $0,16 \pm 0,00109$ & $0,33 \pm 0,0018$ \\
$\%$ Lípidos & $8,74 \pm 0,24$ & $15,76 \pm 0,34$ \\
Concentración Máxima de lípidos $\left./ \mathrm{mg} / \mathrm{L}^{*} \mathrm{~d}\right)$ & $68,172 \pm 1,68$ & $252,16 \pm 2,93$ \\
Productividad lípidos $\left(\mathrm{mg} / \mathrm{L}^{*} \mathrm{~d}\right)$ & $8,52 \pm 0,74$ & $31,52 \pm 2,054$ \\
Productividad biomasa $\left(\mathrm{mg} / \mathrm{L}^{*} \mathrm{~d}\right)$ & $143,85 \pm 7,91$ & $200 \pm 16,83$ \\
Velocidad Máxima Consumo de Glucosa $\left(\mathrm{mg} / \mathrm{L}^{*} \mathrm{~h}\right)$ & $\mathrm{ND}$ & $6,875 \pm 0,603$ \\
$\mathrm{Y}$ x/s con respecto a Glucosa (g biomasa/ g Glucosa) & $\mathrm{ND}$ & $0,7^{*}$ \\
Y p/s Relación de lípidos producidos por glucosa & $\mathrm{ND}$ & 0,18 \\
consumida (g Lípido/ g Glucosa) & & \\
\hline
\end{tabular}

ND. No se determinó el cultivo autotrófico no contenía glucosa *Se calculó con la concentración alcanzada hasta cuando hubo consumo de sustrato.

Se ha reportado que cuando las microalgas se someten a condiciones de estrés impuestas por estímulos ambientales químicos y físicos, solos o en combinación, ocurre síntesis y acumulación de grandes cantidades de lípidos, acompañada por considerables alteraciones en la composición de los lípidos y ácidos grasos [33-35]. En la Tabla 1, se puede apreciar que bajo condiciones de heterotrofia tanto la concentración máxima de lípidos $(252,16 \pm 2,93 \mathrm{mg} / \mathrm{Ld})$ 
como el porcentaje de acumulación de lípidos $(158,7 \pm 3,87 \quad$ mglípidos/gbiomasaseca) fue alrededor de 2 veces mayor en comparación al cultivo autotrófico $(68,172 \pm 1,68 \mathrm{mg} / \mathrm{Ld} \quad \mathrm{y}$ $88,4 \pm 2,63$ mglípidos/gbiomasaseca respectivamente). Diversos autores han reportado que el cultivo heterotrófico permite obtener una mayor acumulación de lípidos. En cultivos de Chlorella sp. se reporta una acumulación de lípidos 3,4 veces mayor que las alcanzadas en autotrofía [20], con Tetraselmis suecica se obtuvo que tanto la productividad como la acumulación de lípidos fue tres veces superior a las condiciones de autotrofía [13], al cultivar células de Chlorella zofingiensis con $30 \mathrm{~g} / \mathrm{L}$ de glucosa en oscuridad se obtuvo un porcentaje dos veces más alto de lípidos totales y una concentración de lípidos de $354 \mathrm{mg} / \mathrm{Ld}$, nueve veces más alta comparada con células el cultivo fotoautotrófico [14].
Los resultados obtenidos en este trabajo resultan congruentes con lo anteriormente expresado ya que las condiciones más favorables para la obtención de lípidos fue en condiciones de heterotrofia donde además se utilizó una concentración de glucosa menor a las reportadas, siendo el valor de $3 \mathrm{~g} / \mathrm{L}$ de glucosa para este trabajo.

\section{Perfil lipídico de la cepa CHL2 en condiciones de heterotrofia}

El contenido y perfil lipídicos de las microalgas son considerados como propios de la especie y no del género, de manera tal que este parámetro varía notablemente entre las especies individuales de cada grupo taxonómico [36]. En la Tabla 2, se muestra el perfil lipídico de CHL2, nótese que el ácido graso predominante es el ácido oleico (18:1) con un $28,75 \%$ del total de ácidos grasos.

Tabla 2. Perfil lipídico de CHL2 en heterotrofia.

\begin{tabular}{ccccc}
\hline Ácido Graso (\%) & CHL2 HETE & Radmann y Vieira (2008) & Sánchez et al. (2012) & Morais y Costa (2008) \\
\hline C 14:0 & 0,35 & ND & ND & 0,5 \\
C 15:0 & 0,09 & 0,14 & ND & ND \\
C 16:0 & 7,24 & 4,36 & 21,59 & 11,6 \\
C 16:1 & 19,75 & 23,47 & ND & 0,4 \\
C 18:0 & 2,04 & 1,20 & 0,52 & 23,0 \\
C 18:1 & 28,75 & 21,81 & 7,67 & 5 \\
C 18:2 & 11,86 & 6,26 & 21,94 & 2,8 \\
r-C 18:3 & 2,61 & 3,12 & 0,17 & 14,1 \\
C 20:0 & 19,37 & 29,10 & ND & 18,9 \\
C 20:1 & 1,21 & 0,37 & ND & 4,2 \\
C 20:3 & 0,84 & 0,57 & ND & 1,4 \\
C 20:5 & 0,76 & ND & ND & 1,8 \\
C 22:0 & 0,94 & ND & ND & 0,7 \\
\hline
\end{tabular}

ND. No se determinó.

Varios autores, han reportado que este ácido graso ejerce una acción beneficiosa en los vasos sanguíneos reduciendo el riesgo de sufrir enfermedades cardiovasculares [37]. Otros ácidos encontrados fueron: ácido palmitoleico (C16:1) 19,75\%, ácido araquídico (C 20:0) 19,37\%, ácido linoleico (C 18:2) 11,86\%, ácido palmítico (C16:0)

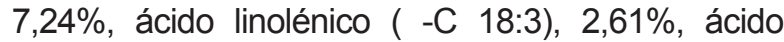
erúcico (C 22:1) 4,61\%, ácido esteárico (C18:0) 2,4\%. Es importante resaltar el ácido palmitoleico, el cual ha sido reportado como un importante constituyente de la dieta humana que ayuda a prevenir enfermedades cardiovasculares [38] y el ácido linoleico es un ácido graso poliinsaturado asociado a la familia de los omega-6, se ha reportado que ayuda a subir las defensas, disminuye los niveles de grasa corporal, disminuye la presión arterial, ayuda a controlar el colesterol y los triglicéridos, reduce el riesgo de enfermedades del sistema circulatorio, ayuda a eliminar las grasas perjudiciales para el organismo, interviene en un buen funcionamiento de los sistemas nervioso y visual [39]. 
El perfil lipídico encontrado en este trabajo está acorde con reportes previos donde utilizando a Chlorella los ácidos predominantes son: palmitoleico, oleico y araquídico [40]. Aunque otros autores reportan que los ácidos grasos que más predominan en cepas de Chlorella son el ácido esteárico, el ácido araquídico [37] y el linoleico [40]. Cabe destacar también que para la cepa $\mathrm{CHL} 2$ se detectaron ácidos grasos poliinsaturados esenciales como el ácido linolénico y el ácido eicosapentaenóico (EPA) en donde este último tiene un valor importante como nutracéutico ya que actúa previniendo la formación de bloques nocivos de prostaglandinas tales como; la prostaglandina D2 (induce la vasodilatación, la hiperalgesia y la quimiotaxis de neutrófilos fuertes); la prostaglandina E2 (induce pirexia, hiperalgesia, la quimio taxis de neutrófilos). El EPA también puede aumentar y mejorar la función inmunológica del cuerpo humano [37].

En la Figura 5 se muestra la asociación de los ácidos grasos de acuerdo al grado de saturación, los ácidos grasos saturados son aquellos que solo poseen enlaces sencillos en la unión de los carbonos que componen la cadena; los monoinsaturados poseen una sola insaturación, es decir posee un solo doble enlace en la cadena de carbonos y finalmente los poliinsaturados que poseen dos o más insaturaciones (dobles enlaces) durante toda la cadena de carbonos.

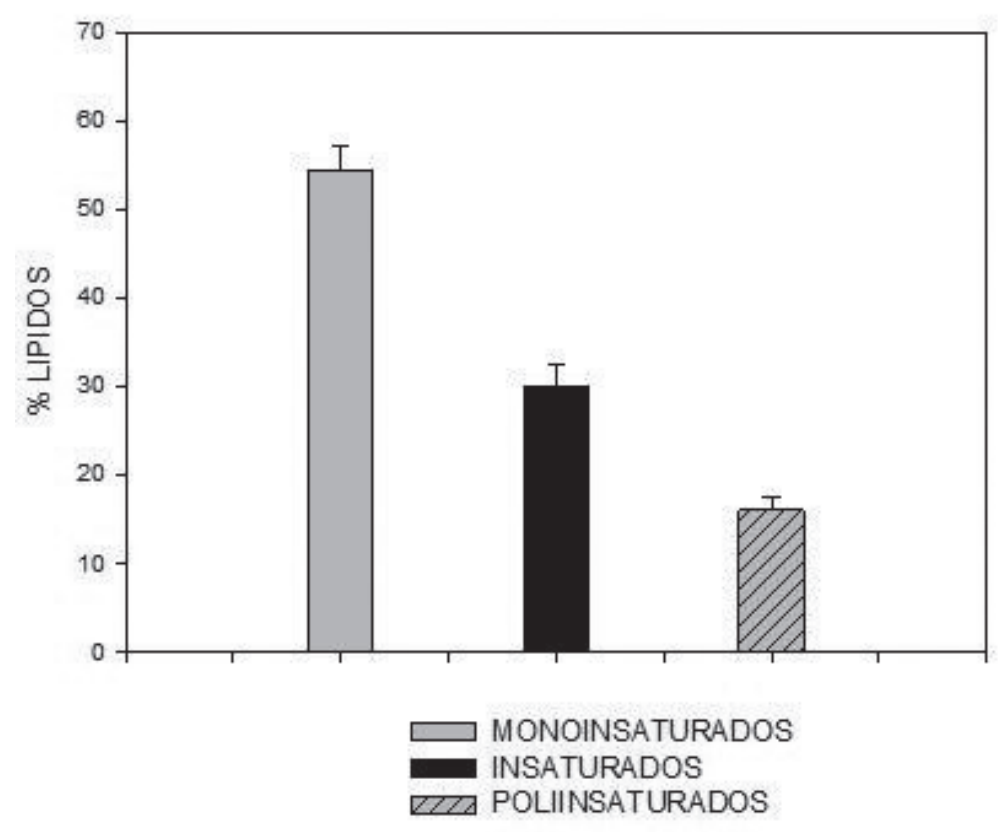

Figura 5. Agrupación de ácidos grasos de acuerdo al grado de saturación.

Como se observa en la Figura 5 para la cepa CHL2 en régimen heterotrófico, el $54 \%$ de los lípidos son monoinsaturados (AGM), el $30,04 \%$ son saturados (AGS) y finalmente el $16,74 \%$ poliinsaturados (AGP). Según reportes de otros autores, para Chlorella vulgaris el porcentaje de ácidos grasos saturados puede oscilar entre $20 \%$ - 30\%, mientras que los poliinsaturados (AGP) pueden oscilar del 5\%-24\% [40]; para cepas de Chlorella un porcentaje de AGS del $40 \%$ y $60 \%$ de AGI [40] y otros reportan $58,60 \%$ de AGS, $15 \%$ de AGM y $26,4 \%$ de AGP [41]. Al comparar los resultados obtenidos con los reportados en la literatura se puede observar que estos son similares, cabe resaltar que el porcentaje de AGP en este trabajo fue mayor que los ya reportados.

Los PUFA's tienen un nivel de importancia alto, pueden servir en la prevención y tratamiento de muchas enfermedades cardiovasculares; reducir la presión sanguínea, los niveles de colesterol en sangre y probabilidad de padecer cáncer, entre otras enfermedades. Actualmente se ha reportado que ayuda a la calidad de vida de pacientes con VIH. La biomasa de microalgas en comparación con otras fuentes de ácidos grasos, tales como varias especies de pescado (bacalao, anchoas y sardinas), presenta algunas ventajas como la ausencia de contaminación con metales pesados 
y ciertas microalgas aún tenían significativamente mayor espectro de PUFA, algunos con cadenas de más de 18 átomos de carbono [40]. Finalmente, se puede observar que el cultivo heterotrófico resulta promisorio para la obtención de PUFA's o AGP, ya que la cepa estudiada contiene un perfil lipídico donde la cantidad de ácidos grasos insaturados son alrededor del $70 \%$, cabe resaltar que en este trabajo no se utilizó concentraciones altas de glucosa, ni se utilizaron limitaciones de nutrientes (fósforo o nitrógeno) que podrían inducir aún más, la producción de PUFA's.

\section{Conclusiones}

Las cepas de Chlorella sp. (CHL2) y Scenedesmus sp. (SCE) se adaptaron a las condiciones heterotróficas. La cepa CHL2 presentó mejores resultados en términos de biomasa $(1,2 \pm 0,014 \mathrm{~g} / \mathrm{L})$ y lípidos (150,4 $\pm 2,3$ mglípidos/gbiomasaseca) en comparación a la cepa SCE $(0,68 \pm 0,02 \mathrm{~g} / \mathrm{L}$ y $62,4 \pm 0,823 \mathrm{mglípidos} / g b i o m a s a s e c a)$, lo anterior permitió seleccionar a la cepa CHL2 para continuar con la producción de PUFA's a escala de 10L. La cinética de 10L de CHL2 permitió obtener una concentración máxima de biomasa de $1,6 \mathrm{~g} / \mathrm{L}$ y una acumulación de lípidos de 157,4mglípidos/gbiomasaseca, los cuales fueron dos veces mayores a los resultados obtenidos por el control en condiciones de autotrofía.

En términos cinéticos se observó que tanto la tasa de crecimiento como la productividad de biomasa y lípidos fue aproximadamente el doble en comparación con el cultivo autotrófico.

El perfil lipídico permitió determinar que el mayor ácido graso presente en CHL2 es el ácido oleico (18:1) con un $28,75 \%$ del total de ácidos grasos, también se destacó la acumulación de: ácido palmitoleico (C 16:1) 19,75\%, ácido araquídico (C 20:0) 19,37\%, ácido linoleico (C 18:2) 11,86\%, ácido palmítico (C 16:0) 7,24\%, ácido linolénico ( $\gamma$-C 18:3), 2,61\%, ácido erúcico (C 22:1) 4,61\%, ácido esteárico (C 18:0) 2,4\%.

La clasificación de los ácidos grasos obtenidos de acuerdo al grado de saturación para la cepa $\mathrm{CHL} 2$ en régimen heterotrófico fue de un $54 \%$ monoinsaturados (AGM), 30,04\% saturados (AGS) y $16,74 \%$ poliinsaturados (AGP o PUFA's).

\section{Agradecimientos}

Los Autores agradecen al Fondo de Investigaciones Universitarias (FINU) de la Universidad Francisco de Paula Santander por la financiación del proyecto.

\section{Referencias bibliográficas}

[1] Herrera M, Vega y León S, Gutiérrez R, García $B$, Díaz G. Los ácidos grasos omega-3 y omega-6: Nutrición, bioquímica y Salud. REB. 2006;25(3):72-9.

[2] Robles A, Molina E, Giménez A, Ibañes MJ. Downstream processing of algal polyunsaturated fatty acids. Biotechnol Adv. 1998;16(3):517-80.

[3] Wen ZY, Chen F. Heterotrophic production of eicosapentaenoid acid by the diatom Nitzschia laevis: effects of silicate and glucose. J Ind Microbiol Biotech. 2000;25:218.

[4] Sforza E, Bertucco A, Morosinotto T, Giacometti GM. Vegetal oil from microalgae: species selection and optimization of growth parameters. Chem. Eng. Trans. 2010;20:199204.

[5] Ratledge C, Gunstone FD. Microorganisms as source of polyunsaturated fatty acids in: structured and modified lipids. Ed. New York: Marcel Dekker. 2001.

[6] Cobos Ruiz M, Paredes Rodríguez JD, Castro Gómez JC. Inducción de la producción de lípidos totales en microalgas sometidas a estrés nutritivo. Acta biol. Colomb. 2016; 21(1):17-26.

[7] Perez García O, Escalante F, De Bashan L, Bashan Y. Heterotrophic cultures of microalgae: metabolism and potential products. Water research. 2011;(45):11-36.

[8] Jaimes D, Soler W, Velasco J, Muñoz Y, Urbina $\mathrm{N}$. Bioprospecting chlorophytas microalgae with potential for the production of lipids for biofuels. CT\&F. 2012;5(1):93-102.

[9] Perales Vela H, González S, Montes MC, Cañizares RO. Growth photosynthetic and respiratory responses to sub-lethal copper concentrations in Scenedesmus incrasatulus (Clorophyceae). Chemosphere. 2007; 67:2274-81. 
[10]Urbina Suarez N. Cultivo mixotrófico de Scenedesmus incrassatulus para la producción de carotenoides en un fotobiorreactor multitubular (tesis de maestría). Distrito Federal, México: Centro de Investigación y de Estudios Avanzados del Instituto Politécnico Nacional - CINVESTAV; 2010.

[11] APHA, AWWA, WPCF. Standard Methods for the Examination of Water and Wastewater. 18a. Edition. E.U.A. 1992

[12] Sánchez Y, Gallo A, Gómez L, Álvarez I, Sabina L, Támbara Y, et. al. Productividad de lípidos y composición de ácidos grasos de cinco especies de microalgas. Investigación y Saberes. 2012;1(2):37-43.

[13] Azma M, Mohamed M, Mohamad R, Rahim R, Ariff A. Improvement of medium composition for heterotrophic cultivation of green microalgae, Tetraselmis suecica, using response surface methodology. Biochem Eng J. 2011;53(2):18795.

[14] Liu J, Huang J, Sun Z, Zhong Y, Jiang Y, Chen F. Differential lipid and fatty acid profiles of photoautotrophic and heterotrophic Chlorella zofingiensis: Assessment of algal oils for biodiesel production. Bioresour Technol. 2011; 102(1):106-10.

[15] Xiong W, Li X, Xiang J, Wu Q. Highdensity fermentation of microalga Chlorella protothecoides in bioreactor for microbio-diesel production. Appl Microbiol Biotechnol. 2008; 78(1):29-36.

[16] Shen Y, Yuan W, Mao E. Heterotrophic Culture of Chlorella protothecoides in Various Nitrogen Sources for Lipid Production, Appl. Biochem. Biotechnol. 2010;160(6):1674-84.

[17] Arias M, Martínez A, Cañizares R. Producción de biodiesel a partir de microalgas: parámetros del cultivo que afectan la producción de lípidos. Acta Biol. Colomb. 2013;18 (1):43-68.

[18] Miao X, Wu Q. High yield bio-oil production from fast pyrolysis by metabolic controlling of Chlorella protothecoides. J Biotechnol. 2004; 110(1):85-93.

[19] Miao X, Wu Q. Biodiesel production from heterotrophic microalgal oil. Bioresour Technol. 2006;97(6):841-6.

[20] Xu H, Miao X, Wu Q. High quality biodiesel production from a microalga Chlorella protothecoides by heterotrophic growth in fermenters. J Biotechnol. 2006;126(4):499-507.

[21] Zheng Y, Chi Z, Lucker B, Chen S. Two-stage heterotrophic and phototrophic culture strategy for algal biomass and lipid production. Bioresour Technol. 2012;103(1):484-8.

[22] Jaimes D, Soler W. Producción de lípidos a partir de microalgas nativas de la división Chlorophyta de Norte de Santander utilizando aguas residuales (tesis de pregrado). Cúcuta, Colombia: Universidad Francisco de Paula Santander; 2013.

[23] Bouaraba L, DautabA, Loudikia M. Heterotrophic and mixotrophic growth of Micractinium pusillum Fresenius in the presence of acetate and glucose: effect of light and acetate gradient concentration. Wat Res. 2004;38:2706-12.

[24] Servin-Reyssac J, De la Nouë J, Proulx D. Le recyclage du lisier de porc par lagunage. Edit. Lavoisier editor, Technique y documentation. 1995.

[25] Cheirsilp B, Torpee S. Enhanced growth and lipid production of microalgae under mixotrophic culture condition: Effect of light intensity, glucose concentration and fed-batch cultivation. Bioresour Technol. 2012;110:510-6.

[26] Liang Y, Sarkany N, Cui Y. Biomass and lipid productivities of Chlorella vulgaris under autotrophic, heterotrophic and mixotrophic growth conditions. Biotechnol Lett. 2009;31(7):1043-9.

[27] Fernández D, Chica C, Parra M. Obtención de ácidos grasos a partir de biomasa microalgal cultivada bajo diferentes condiciones de iluminación. Rev. Elementos. 2013;(3):111-9.

[28] García JL, Molina E, García F, Sánchez JA, Giménez A. Cuantificación de ácidos grasos a partir de biomasa microalgal. Grasas y Aceites. 1993;44(6):348-53.

[29] Chisti Y. Biodiesel form Microalgae. Biotech Adv. 2007;25:294-306.

[30] Rosenberg JN, Oyler GA, Wilkinson L, Betenbaugh MJ. A green light for engineered algae: redirecting metabolism to fuel a biotechnology revolution. Curr. Opin. Biotechnol. 2008;19:430-6.

[31] Jacob-Lopes E, Gimenes C, Ferreira L, Teixeira T. Effect of light cycles (night/day) on $\mathrm{CO}_{2}$ fixation and biomass production by microalgae in photobioreactors. Chem. Eng. and Proc. 2009;48:306-10.

[32] Mérida LGR, Zepka LQ, Jacob-Lopes E. Fotobiorreactor: Herramienta para cultivo de cianobacterias. Ciencia y Tecnología. 2013; 6(2):9-19.

[33] De BK, Chaudhury S, Bhattacharyya DK. Effect of nitrogen sources on $y$-linoleic acid 
accumulation in Spirulina platensis. Journ. American Oil Chemists' Soc. 1999;76(1):153-6

[34] Li Q, Du W, Liu D. Perspectives of microbial oils for biodiesel production. Appl. Microbiol. Biotechnol. 2008;80(5):749-56.

[35] Hu Q, Sommerfeld M, Jarvis E, Ghirardi M, Posewitz M, Seibert M, et. al. Microalgal triacylglycerols as feedstock for biofuel production: perspectives and advances. Plant J. 2008;54(4):621-39.

[36] Montero Y, Gallo A, Gómez L, Álvarez I, Sabina L, Támbara $Y$, et. al. Productividad de lípidos y composición de ácidos grasos de cinco especies de microalgas. Investigación y Saberes. 2012;1(2):37-43.

[37] Greque de Morais M, Vieira J. Fatty acids profile of microalgae cultived with carbon dioxide. Ciênc. agrotec. 2008;32(4):1245-51.

[38] Willis WM, Lencki RE, Marangoni AG. Lipid modification strategies in the production of nutritionally functional fats and oils. Crit. Rev. Food Sci.1998;38(8):639-74.

[39] Castro M. Ácidos grasos omega 3: beneficios y fuentes. Interciencia. 2002;27(3):128-36.

[40]Radmann M, Viera J. Conteúdo lipídico e composição de ácidos graxos de microalgas expostas aos gases $\mathrm{CO}_{2}, \mathrm{SO}_{2}$ e $\mathrm{NO}$. Quim. Nova. 2008;31(7):1609-12.

[41] Muradyan EA, Klyachko-Gurvich GL, Tsoglin L, Sergeyenko T, Pronina NA. Changes in Lipid Metabolism during Adaptation of the Dunaliella salina Photosynthetic Apparatus to High $\mathrm{CO}_{2}$ Concentration Russ. J. Plant Physiol. 2004; 51:53-62. 